\title{
A DUAL-PIVOT PATTERN SIMULATING NATIVE KNEE KINEMATICS OPTIMIZES FUNCTIONAL OUTCOMES AFTER TOTAL KNEE ARTHROPLASTY
}

R. Michael Meneghini, MD ${ }^{1,2}$

Evan R. Deckard, BS ${ }^{2}$

Marshall K. Ishmael, BS ${ }^{2}$

Mary Ziemba-Davis, BA $^{2}$

${ }^{1}$ Indiana University School of Medicine, Department of Orthopaedic Surgery, 1120 W. Michigan Street, Room 600, Indianapolis, IN 46202

${ }^{2}$ Indiana University Health Physicians Orthopedics and Sports Medicine, IU Health Saxony Hospital, 13100 East $136^{\text {th }}$ Street, Suite 2000, Fishers, IN 46037

Corresponding Author:

R. Michael Meneghini, MD

Indiana University Health Physicians Orthopedics and Sports Medicine

Indiana University School of Medicine, Department of Orthopaedic Surgery

$13100136^{\text {th }}$ Street

Suite 2000

Fishers, IN 46037

Phone: 317-688-5980

rmeneghi@iuhealth.org

This is the author's manuscript of the article published in final edited form as:

Meneghini, R. M., Deckard, E. R., Ishmael, M. K., \& Ziemba-Davis, M. (2017). A Dual-Pivot Pattern

Simulating Native Knee Kinematics Optimizes Functional Outcomes After Total Knee Arthroplasty. The 
A DUAL-PIVOT PATTERN SIMULATING NATIVE KNEE KINEMATICS OPTIMIZES FUNCTIONAL OUTCOMES AFTER TOTAL KNEE ARTHROPLASTY

\section{Abstract}

7 Background: Kinematics after total knee arthroplasty (TKA) have been studied for decades;

8 however, few studies have correlated kinematic patterns to patient reported outcomes. The

9 purpose of this study was to determine if a pattern of lateral pivot motion in early flexion and medial pivot motion in high flexion, simulating native knee kinematics, produces superior

11 clinical outcomes. A second study objective was to determine if a specific kinematic pattern 12 throughout the various ranges of flexion produces superior function and patient satisfaction.

13 Methods: 120 consecutive TKAs were performed using sensor embedded tibial trials to 14 record intraoperative knee kinematics through the full range of motion. Established criteria were used to identify lateral (L) or medial (M) pivot kinematic patterns based on the center of rotation within three flexion zones -- 0 to $45^{\circ}$ (early flexion), 45 to $90^{\circ}$ (mid flexion) and $90^{\circ}$

17 to terminal flexion (late flexion). Knee Society Scores, pain scores, and patient satisfaction 18 were analysed in relationship to kinematic patterns.

19 Results: Knee Society function scores were significantly higher in TKAs with early lateral 20 pivot/late medial pivot intraoperative kinematics compared to all other kinematic patterns ( $p$ $21=0.018)$ at minimum one-year follow-up. There was a greater decrease in the proportion of 22 patients with early lateral/late medial pivot kinematics who reported that their knee never 23 feels normal $(p=0.011)$. Higher mean function scores at minimum one-year follow-up $(p<$ $240.001)$ and improvement from preoperative baseline $(p=0.008)$ were observed in patients with the most ideal "LLM" kinematic pattern (lateral pivot 0 to $45^{\circ}$ and 45 to $90^{\circ}$; medial 26 pivot beyond $90^{\circ}$ ) compared to those with the least ideal "MLL" kinematic pattern. All 
27 patients with the optimal "LLM" kinematic pattern compared to none of those with the

28 "MLL" kinematic pattern reported that they were very satisfied with their TKA $(p=0.003)$.

29 Conclusion: Patients who exhibited an early flexion lateral pivot kinematic pattern

30 accompanied by medial pivot motion in later flexion, as measured intraoperatively, reported

31 higher functional outcome scores along with higher overall patient satisfaction. Replicating

32 the dual-pivot kinematic pattern observed in native knees may improve function and

33 satisfaction after TKA. Further study is warranted to explore a correlation with in-vivo

34 kinematic patterns.

35 Keywords: total knee arthroplasty, kinematics, patient reported outcomes

36

37

38 


\section{Introduction}

Total knee arthroplasty (TKA) is exceptionally reliable in terms of implant longevity and survivorship; however, patient reported outcomes after TKA reveal the disappointing fact that up to $20 \%$ of patients are not satisfied, [1] often with continued pain, stiffness, or an 'unnatural' feel to the joint. Knee kinematics, which detail the tibiofemoral contact locations and movement patterns of the knee, have been studied for decades and are postulated to correlate with clinical outcomes after TKA. Further, it has been hypothesized that knee arthroplasty systems that replicate kinematic patterns of the native knee with an intact anterior cruciate ligament (ACL), particularly unicompartmental and bicruciate-preserving knee arthroplasty, will reproduce normal knee motion and potentially optimize patient function, outcomes, and satisfaction after TKA. While various implant designs and types have been studied with respect to kinematic patterns, [2-14] the search continues for clinical evidence to support one kinematic pattern over another in producing superior patient outcomes.

Traditional understanding of native knee kinematics has supported a medial-pivot kinematic pattern throughout the entire knee range of motion. [15-18] Since 2008, a more modern understanding of native knee kinematics has revealed a more complex kinematic pattern of differing pivot motions in the various flexion ranges within the full knee range of motion. [19-23] While modern kinematics continue to support a medial pivot tibiofemoral contact pattern with deeper flexion activities in the native knee, it is now understood that native knee kinematics in earlier flexion angles occurring with activities like walking, running, or pivoting are characterized by a lateral pivot pattern. [20-23] Sensor-embedded tibial trials have been developed to provide real-time intraoperative tibiofemoral contact forces to objectively quantify soft tissue balance during TKA procedures. [24, 25] Sensorembedded tibial inserts visually locate and characterize the kinematic femoral contact points 
64 on the tibia intraoperatively. The purpose of this study was to determine if an intraoperative pattern of lateral pivot motion in early flexion $\left(0\right.$ to $\left.45^{\circ}\right)$ and medial pivot motion in late flexion $\left(90^{\circ}\right.$ to terminal flexion), simulating native knee kinematics, produces superior patient-reported outcomes compared to other kinematic patterns. A second objective of this study was to determine if a specific kinematic pattern, designated as medial or lateral pivot at the various flexion angle ranges of 0 to $45^{\circ}, 45$ to $90^{\circ}$, and $90^{\circ}$ to terminal flexion, produces superior patient-reported outcomes after TKA.

\section{Methods}

With institutional review board approval, a retrospective review of a prospectively collected database of 120 consecutive primary TKAs was undertaken. Procedures were performed between April 2013 and April 2014 by two board-certified, high volume arthroplasty surgeons at a single institution. All patients presenting for a primary TKA for a diagnosis of osteoarthritis or autoimmune associated knee arthritis were included. In each case, sensor-embedded tibial trials $\left(\right.$ Verasense $^{\mathrm{TM}}$, OrthoSensor ${ }^{\mathrm{TM}}$, Sunrise, FL) were used to track tibiofemoral contact points following TKA implantation using traditional balancing techniques based on manual and tactile surgeon judgment. The balancing technique utilized is a measured resection technique with diligent assessment of gap balance with spacer blocks or calibrated lamina spreaders and fine-tuning with soft-tissue balancing after bone resection cuts were made. Thirty-four TKAs were excluded to eliminate potential bias for the following reasons: unavailability of the required size of the Verasense ${ }^{\mathrm{TM}}$ device $(n=16)$, device malfunction $(n=5)$, atypical hardware creating additional soft tissue trauma $(n=5)$, surgery performed at a non-study hospital without the availability of the Verasense ${ }^{\mathrm{TM}}$ insert trials $(n=4)$, unresurfaced patella $(n=1)$, early revision $(n=2$; one for infection and one for

87 tibial aseptic loosening), and death unrelated to the index TKA $(n=1)$. Of the remaining 86 
88 TKAs, seven $(8.1 \%)$ were lost to minimum one-year follow-up, resulting in a sample size of 79 TKAs.

A median parapatellar approach was used for all procedures. Standard coronal plane

tibial and femoral bone cuts were made with computer-aided navigation (Stryker Navigation, Kalamazoo, MI). One knee arthroplasty system (Triathlon®, Stryker, Inc., Mahwah, NJ) was used in all patients. One surgeon routinely retained the posterior cruciate ligament (PCL) and utilized a cruciate-retaining (CR) implant with a CR or a cruciate stabilizing (CS) insert with an anterior lip. The other surgeon routinely sacrificed the PCL and used a CS insert with an anterior lip. Posteriorly-stabilized implants were not used in study TKAs.

Verasense ${ }^{\mathrm{TM}}$ data were acquired once the final implants were in place and the retinaculum was closed to most accurately measure intraoperative contact forces and kinematic patterns throughout the range of motion as has been described previously by numerous authors. [26-29] Tibiofemoral contact points were recorded for each patient at terminal extension $\left(0^{\circ}\right)$, at $45^{\circ}$ and $90^{\circ}$ of flexion, and at terminal flexion. Patient age, sex,

102 body mass index (BMI), and surgeon were recorded.

\section{Data Extraction}

The Verasense ${ }^{\mathrm{TM}}$ device produces images of tibiofemoral contact locations within triangular areas representing the medial and lateral tibial plateau surfaces as the knee is moved through the range of motion intraoperatively (Figure 1). Four static images per

107 patient were cropped from the continuous Verasense ${ }^{\mathrm{TM}}$ video and graphic user interface feed, 108 one each for the knee at $0^{\circ}, 45^{\circ}, 90^{\circ}$, and terminal flexion (Figure 2). The cropped images were imported into MATLAB® (The Mathworks, Natick, MA) after alterations were conducted in Microsoft Paint ${ }^{\circledR}$ (Microsoft, Redmond, WA) to determine the exact position of

111 the contact points using a custom image processing program. The custom image processing 112 program operated based on detecting color differences within the cropped images to isolate 
113 the coloured dots associated with the medial and lateral tibiofemoral contact locations.

114 Potential error in calculations by MATLAB® was eliminated by "blacking out" all

115 unnecessary color from the image. The only remaining items from the original cropped image

116 were the contact points and the universal origin explained below (Figure 2).

117 Verasense ${ }^{\mathrm{TM}}$ device images uniformly had an "embossed" circle at the center of each

118 tibial surface image standardly produced and located in manufacturing. On each image, we

119 placed a white dot in these circles to create a universal origin for all measurements (Figure 2).

120 This universal origin was determined based on the center of the tibial sensor trial and

121 remained constant throughout data extraction for each patient and different implant sizes.

122 The centroid of each isolated tibiofemoral contact point was calculated with built-in

123 MATLAB® commands from the image processing toolbox. Each image was appropriately

124 scaled based on the screen resolution and screen size from which the image was cropped. The

125 delta values between the contact points and the universal origin were then calculated and

126 exported to an Excel (Microsoft Corporation, Redmond, WA) spreadsheet for further

127 analyses via MATLAB®. Medial and lateral tibiofemoral contact points at each range of

128 motion were connected by lines (Figure 3 ) to permit calculation of centers of rotation

129 (CORS) as the intersection points of two lines at different ranges of motion (e.g., the

130 intersection of the line associated with medial-lateral contact points at $0^{\circ}$ and the same line at

$\left.13145^{\circ}\right)$. CORS were calculated based on vectors for early flexion $\left(0\right.$ to $\left.45^{\circ}\right)$, mid-flexion $\left(45^{\circ}\right.$ to

$\left.13290^{\circ}\right)$ and late flexion $\left(90^{\circ}\right.$ to terminal). COR values were then used to determine if the

133 kinematic pattern between the two flexion angles was medial or lateral based on their

134 location with reference to the medial and lateral compartments. If the COR was located in the

135 medial compartment between $5 \mathrm{~mm}$ and $1000 \mathrm{~mm}$, the kinematic pattern was determined to

136 be a medial pivot knee between the two distinct flexion angles. If the COR was located in the

137 lateral compartment between $-5 \mathrm{~mm}$ and $-1000 \mathrm{~mm}$, the kinematic pattern was determined to 
138 be a lateral pivot knee between the two distinct flexion angles If the COR was less than 5 or

139 greater than $-5 \mathrm{~mm}$, it was considered a central pivot. If the COR was greater than $1000 \mathrm{~mm}$

140 or less than $-1000 \mathrm{~mm}$, it was considered a translation of the implant due to the COR value

141 not allowing a detectable pivot pattern and therefore sliding instead of rotating.

142 Study Groups:

143 To address the first study question (whether an intraoperative pattern of lateral pivot

144 motion in early flexion and medial pivot motion in late flexion produces superior patient-

145 reported outcomes), patients were placed into two distinct kinematic pattern groups. The first

146 group ("early lateral/late medial pivot group") included those TKAs with a lateral pivot in

147 early flexion $\left(0\right.$ to $\left.45^{\circ}\right)$ and a medial pivot in late flexion $\left(90^{\circ}\right.$ to terminal flexion), simulating

148 the kinematic pattern of the native ACL-intact knee. The second group ("other kinematic

149 patterns group") included TKAs exhibiting all other patterns not included in the first group,

150 which by definition included knees with any kinematic pivot (lateral or medial) other than

151 lateral pivot from 0 to $45^{\circ}$ and medial pivot from $90^{\circ}$ to terminal flexion including lateral-

152 lateral, medial-lateral, and medial-medial piyot patterns. Knees with central or translational

153 pivot patterns in early or late flexion were excluded from statistical analyses resulting in

154 samples of 16 early lateral/late medial pivot knees and 47 knees which have been denoted as

155 "other" kinematic patterns as described above and represented graphically in Figures 4 and 5.

156 To address the second study question (whether a specific kinematic pattern produces

157 superior patient-reported outcomes after TKA), the kinematic pattern in three distinct flexion

158 zones -0 to $45^{\circ}$ (early flexion), 45 to $90^{\circ}$ (mid-flexion), and $90^{\circ}$ to terminal flexion (late

159 flexion) — was noted by a three letter designation according to the pattern within each flexion

160 zone. For example, a designation of "LLM" was used to indicate that the TKA

161 intraoperatively demonstrated lateral pivot motion in early flexion, lateral pivot motion in

162 mid-flexion, and medial pivot motion in late flexion. Knees with central or translational pivot 
163 patterns in early, mid-, or late flexion were excluded from statistical analyses. Upon review

164 of Knee Society function scores for all patterns, we proceeded with comparisons of the

165 theoretically and statistically ideal (LLM, $\mathrm{n}=8$ knees) and least ideal (MLL, $\mathrm{n}=6$ knees)

166 kinematic patterns.

167 Patient Reported Outcomes

168 Patient reported outcomes were evaluated preoperatively and at minimum one-year 169 postoperatively utilizing the new Knee Society Scoring (KSS) system. [30, 31] The new

170 KSS system consists of validated objective and subjective scores. The Knee Society objective

171 score, denoted "KSSO" in this manuscript, evaluates knee pain (25 points), alignment (25

172 points), stability (25 points), and range of motion (25 points) for a total possible score of 100.

173 Total possible points for the subjective satisfaction (denoted "KSSS" in this manuscript) and

174 functional (denoted "KSSF" in this manuscript) components of the new Knee Society Score,

175 are 40 points and 100 points, respectively. Individual items from the Knee Society

176 questionnaire, including pain with level walking and pain with stairs or inclines (both scored

$1770=$ none to $10=$ severe) also are reported. In addition, responses to a global question "What

178 is your current level of satisfaction with your knee replacement surgery?" (very satisfied,

179 satisfied, neutral, dissatisfied, very dissatisfied) were analysed. The University of California

180 Los Angeles (UCLA) Activity Level Score [32] asks patients to choose their highest level of

181 current activity, ranging from 0 (Wholly Inactive: dependent upon others, cannot leave

182 residence) to 10 (Regularly participate in impact sports such as jogging, tennis, skiing,

183 acrobatics, ballet, heavy labor, or backpacking).

$184 \quad$ Statistical Analysis

185 Patient reported outcome scores were analysed in relationship to kinematic patterns.

186 Minitab 17 (State College, PA) was used for statistical analysis. Data were evaluated for

187 normality using Anderson-Darling tests. Normally distributed continuous variables were 
188

189

190

191

192

193

194

195

196

197

198

199

200

201

202

203

204

205

206

207

208

209

210

211

212

analysed with Student's two-sample t-test (t) and Analysis of Variance (F) while nonnormally distributed continuous variables were compared with the Mann-Whitney (W) or Kruskal-Wallis $(\mathrm{H})$ tests adjusted for ties. Pearson's Chi-Square $\left(\mathrm{X}^{2}\right)$ test was used to test independence among categorical variables, with Fishers Exact test $p$ values reported for $2 \times 2$ contingency tables. A significance level of 0.05 was used for all statistical analyses.

\section{Results}

Early Lateral Pivot / Late Medial Pivot Group Compared to All Other Kinematic Patterns:

Age, sex, and BMI did not differ between the early lateral pivot/late medial pivot group and the other kinematic patterns group (Table 1). Median follow-up in the former group was shorter by 6.2 months (Table $1, p=0.030$ ). There were no differences in preoperative outcome scores between the two groups (Table 2).

There were $11 \mathrm{CR}$ with CR inserts knees, $34 \mathrm{CR}$ with CS insert knees, and 18 cruciate-sacrificing with CS insert knees. With one exception, outcomes did not vary by implant type ( $p \geq 0.163$ ). Median UCLA Activity Level was 6 in CR/CR knees, 5 in CR/CS knees, and 4 in cruciate-sacrificing/CS knees $(H=6.63, p=0.036)$, reflecting a difference in regular participation in moderate activities such as swimming and unlimited housework or shopping, sometimes participating in these moderate activities, and regular participation in mild activities such as walking, limited housework, or limited shopping, respectively.

At minimum one-year follow-up, mean KSSF scores were significantly higher in TKAs with early lateral pivot/late medial pivot intraoperative kinematics compared to all other kinematic patterns ( 80 vs. $69, \mathrm{t}=-2.51, p=0.018$; Table 2 ). All other clinical outcome scores at minimum one-year follow up did not differ between the two kinematic pattern groups (Table 2).

Improvement from preoperative baseline to minimum one-year outcome scores showed statistical trends for greater improvement in mean KSSF (41.1 vs. 32.2 points, $\mathrm{t}=-$ 
$2131.67, p=0.108)$ and median KSSS (26 vs. 20 points, $\mathrm{W}=1401.5, p=0.107)$ in the early

214 lateral pivot/late medial pivot kinematic pattern group compared to other kinematic patterns 215 group (Table 2).

216 Overall satisfaction with TKA is shown graphically in Figure 4 separately for the

217 early lateral/late medial kinematic pattern group and the other kinematic patterns group.

218 Eighty-six percent of the former group compared to only 57\% of the latter group reported that 219 they were very satisfied with their TKA $\left(X^{2}=3.729, p=0.099\right)$. Figure 5 shows the percent 220 change from preoperative baseline in the proportion of patients in each group who reported

221 that their knee always, sometimes, or never feels normal. While percent change in the 222 proportions of the early lateral/late medial kinematic pattern group and the other kinematic 223 patterns group reporting that their knee always feels normal was not statistically different (a $22456.3 \%$ increase vs. a $47.6 \%$ increase, $\mathrm{t}=1.081, p=0.284)$, there was a significantly greater 225 decrease in the proportion of patients in the former group compared to the latter group who 226 reported that their knee never feels normal (a 50.9\% decrease vs. a 16.7\% decrease, $\mathrm{t}=2.650$, $227 p=0.011)$.

\section{LLM and MLL Kinematic Patterns:}

In this analysis, there were $2 \mathrm{CR}$ with CR inserts knees, $9 \mathrm{CR}$ with CS insert knees, and 3 cruciate-sacrificing with CS insert knees. Outcomes did not vary by implant type ( $p \geq$

231 0.291). Analysis of minimum one-year KSSF function scores $(F=3.80, p=0.004)$ and the 232 amount of improvement in KSSF from preoperative baseline $(\mathrm{F}=1.21, p=0.321)$ suggested 233 a clear distinction in mean functional outcomes scores among all available kinematic patterns 234 based on early, mid-, and late flexion (Figure 6). In particular, as shown in Table 3, patients 235 with the most ideal LLM kinematic pattern had significantly higher mean function scores at 236 minimum one-year follow-up (87.5 vs. 51.2 points, $\mathrm{t}=6.89, p<0.001)$ and improvement 237 from preoperative baseline ( 48.3 vs. 25.7 points, $\mathrm{t}=3.26, p=0.008$ ) than patients with the 
238 least ideal MLL kinematic pattern. Table 3 also shows that patients with an LLM kinematic

239 pattern compared to those with the MLL pattern were significantly more satisfied with their

240 TKA as measured by KSSS at minimum one-year follow-up (medians of 40 vs. 33 points, W

$241=75.5, p=0.043$ ) and improvement in KSSS from baseline (mean improvements of 27.5 and

24218 points, $\mathrm{t}=2.68, p=0.022$ ).

243 As shown in Figure 7, all patients with an intraoperative LLM kinematic pattern in

244 early, mid-, and late flexion ( $\mathrm{n}=8$ knees) compared to none of the patients with the MLL

245 kinematic pattern $(n=6$ knees) reported that they were very satisfied with their TKA at

246 minimum one-year follow-up $\left(\mathrm{X}^{2}=11.0, p=0.003\right)$.

\section{Discussion}

248 Kinematic patterns in TKA have been extensively studied to date; [2-14, 33] however,

249 the search continues for clinical evidence to support one kinematic pattern over another in

250 producing superior patient outcomes. Dennis and co-authors published a comprehensive

251 kinematic analysis of 811 TKAs of numerous designs, from multiple institutions and

252 surgeons, and reported that substantial variability occurred in all designs and groupings with

253 respect to kinematic patterns. [33] Further, the authors reported that a desirable medial pivot

254 pattern in flexion was present in only $55 \%$ of TKAs in the analysis, suggesting that as

255 surgeons we have little ability to reliably induce a particular kinematic pivot pattern in TKA.

256 This variability in kinematic patterns observed in modern TKA and the inability to reproduce

257 an ideal target kinematic pattern may contribute to the reported 15 to $20 \%$ of TKA patients

258 who are not satisfied with their TKA. [1]

Traditionally, understanding of native knee kinematics has supported a medial pivot

260 kinematic pattern throughout the entire range of knee flexion. [15-18] In 2003, Komistek and

261 co-authors [17] published an elegant fluoroscopic study on five native knees and reported

262 predominantly medial pivot kinematic patterns throughout flexion on average in the five 
subjects. However, the authors also observed that substantially less tibial rotation occurred in gait $(<5$ degrees) when compared to greater flexion activities such as a deep knee bend $(<13$ degrees) and one of the knees demonstrated a lateral pivot motion in gait and deeper flexion.

266 Since 2008, a more modern understanding of native knee kinematics has revealed a more 267 complex kinematic pattern of differing pivot motions in the various knee flexion ranges. [20-

268 23] While modern kinematics continues to support a medial pivot pattern with deeper flexion activities, it is now understood that native knee motion in earlier flexion angles, occurring with activities like walking, running or pivoting, are characterized by a lateral pivot pattern.

272 patients specifically with regard to walking. Using a point-cluster gait analysis technique, it

273 was demonstrated that the center of rotation during the stance phase of walking was in the lateral compartment for all 46 knees. In addition, the instantaneous center of rotation occurred on the medial side on average less than $25 \%$ of the time during the stance phase.

276 Further supporting this notion, Hoshino and Tashman [19] reported the kinematic

277 tibiofemoral contact patterns of 29 native knees during downhill running. The authors

278 utilized three dimensional CT scans and dynamic bi-planar fluoroscopy and discovered that 279 the sliding contact path of the femur on the tibia was significantly greater on the medial side compared to the lateral side, suggesting that lateral pivot kinematic pattern is present during running. These studies support the evolution of knee kinematics in the ACL-intact native

282 knee to an understanding that in early flexion activities, such as walking and running, the 283 dominant pattern is lateral pivot motion, while the traditional medial pivot pattern continues to predominate in deeper flexion activities. contact forces to objectively quantify soft tissue balance during a TKA procedure. [24, 25]

287 The sensor-embedded tibial inserts also visually locate and characterize the kinematic 
femoral contact points on the tibia, which can provide intraoperative kinematic pattern data acquisition in real-time. Our findings suggest that patients who intraoperatively exhibit the early flexion lateral pivot pattern and late flexion medial pivot kinematic pattern possess

higher overall satisfaction with their knee replacement surgery as well as an improvement with the function of their knee as measured by modern Knee Society Function scores. When defining the kinematic pattern in a more complex manner utilizing the patterns in all three flexion ranges, patient reported outcome scores of the "LLM" kinematic pattern (lateral pivot pattern in 0 to $45^{\circ}$ and 45 to $90^{\circ}$ degree ranges and medial pivot in the high flexion range beyond $90^{\circ}$ ) suggest this pattern to be the best overall in terms of satisfaction and function.

297 Conversely, the kinematic pattern identified as the worst kinematic pattern to experience was 298 the exact opposite pattern "MLL", further supporting the optimal outcomes are potentially more likely if kinematic patterns exist in TKAs that replicate the native knee kinematics with an intact ACL. While "LLM" was the optimal pattern observed in this data analysis, the midflexion zone of 45 to $90^{\circ}$ flexion remains to be further studied, as the ACL-intact native knee studies referenced above are non-specific and variable with respect to the exact flexion point where the pattern switches from lateral pivot in early flexion to medial pivot in greater flexion, and likely varies among individual patients. intraoperatively during non-weight bearing conditions with a patient anesthetized and may not represent the actual kinematic patterns observed in-vivo during weight bearing through

308 the range of flexion described. However, there is some support that intraoperative 309 measurements of force and balance obtained with intraoperative sensors, can predict in-vivo 310 kinematic patterns. [34] This is certainly an area of further study to determine if a correlation 311 exists between kinematic patterns obtained during surgery and those exhibited in-vivo during 312 weight-bearing functional activities. Second, sensor-embedded tibial trial inserts have not 
313 been validated as measurements of tibiofemoral contact patterns and thus, this study

314 represents the first to utilize this technology for kinematic motion intraoperatively. Finally,

315 due to the relatively small numbers of patients in kinematic pattern groups based on all three

316 flexion ranges, non-significant study results may be attributable to insufficient statistical

317 power. Power for non-significant findings ranged from $<10 \%$ to $90.6 \%$. Further

318 confounding this issue is the inclusion of both cruciate-substituting and cruciate-sacrificing

319 TKA designs of both varus and valgus alignments, which ultimately could affect kinematic

320 patterns in-vivo. However, based on previous kinematic studies which traditionally have

321 relatively small numbers, the authors believe this work provides valuable information for

322 consideration in future research on knee kinematics following TKA. Further, our analysis

323 utilized the modern Knee Society Score which has been validated to more aptly discern a

324 patient's ability to perform various functional activities compared to previous generations of

325 less robust outcome measures. The authors are unaware of any published study that

326 correlates kinematic data and modern Knee Society outcome scores in patients undergoing

327 primary TKA.

328 Based on modern understanding of the dual-pivot kinematic pattern observed in the

329 native ACL-intact knee, more appropriate analysis can be performed regarding TKA

330 kinematics and their correlation with clinical outcomes. It appears that patients who exhibit

331 an early flexion lateral pivot kinematic pattern accompanied by medial pivot motion in late

332 flexion, as measured intraoperatively, may have higher functional outcome scores along with

333 higher overall patient satisfaction. Therefore, replicating the dual-pivot kinematic pattern

334 observed in native knees may improve function and satisfaction after TKA. Further work to

335 identify the extent to which intraoperative kinematic patterns are correlated with in-vivo

336 weight bearing kinematic patterns is necessary. In addition, investigation into the various

337 characteristics of patient anatomy, implant alignment and design, ligament balance, and 
338 surgical technique that might facilitate a kinematic pattern more closely approximating the 339 native knee is warranted.

340 
References

1. Dunbar MJ, Richardson G, Robertsson O. I can't get no satisfaction after my total knee replacement: rhymes and reasons. The bone \& joint journal 95-b(11 Suppl A): 148, 2013 2. Banks SA, Markovich GD, Hodge WA. In vivo kinematics of cruciate-retaining and -

345 substituting knee arthroplasties. The Journal of arthroplasty 12(3): 297, 1997

346 3. Banks SA, Hodge WA. Implant design affects knee arthroplasty kinematics during stair-

347 stepping. Clinical orthopaedics and related research (426): 187, 2004

348 4. Banks SA, Hodge WA. 2003 Hap Paul Award Paper of the International Society for

349 Technology in Arthroplasty. Design and activity dependence of kinematics in fixed and

350 mobile-bearing knee arthroplasties. The Journal of arthroplasty 19(7): 809, 2004

351 5. Bellemans J, Banks S, Victor J, Vandenneucker H, Moemans A. Fluoroscopic analysis of

352 the kinematics of deep flexion in total knee arthroplasty. Influence of posterior condylar

353 offset. The Journal of bone and joint surgery British volume 84(1): 50, 2002

354 6. Chouteau J, Lerat JL, Testa R, Moyen B, Banks SA. Sagittal laxity after posterior cruciate 355 ligament-retaining mobile-bearing total knee arthroplasty. The Journal of arthroplasty 24(5):

356710,2009

357 7. Chouteau J, Lerat JL, Testa R, Moyen B, Fessy MH, Banks SA. Kinematics of a 358 cementless mobile bearing posterior cruciate ligament-retaining total knee arthroplasty. The 359 Knee 16(3): 223, 2009

360 8. Coughlin KM, Incavo SJ, Doohen RR, Gamada K, Banks S, Beynnon BD. Kneeling 361 kinematics after total knee arthroplasty: anterior-posterior contact position of a standard and a 362 high-flex tibial insert design. The Journal of arthroplasty 22(2): 160, 2007

363 9. Dennis DA, Komistek RD, Mahfouz MR. In vivo fluoroscopic analysis of fixed-bearing 364 total knee replacements. Clinical orthopaedics and related research (410): 114, 2003 
365 10. Goodfellow J, O'Connor J. The mechanics of the knee and prosthesis design. The Journal

366 of bone and joint surgery British volume 60-b(3): 358, 1978

367 11. Kanekasu K, Banks SA, Honjo S, Nakata O, Kato H. Fluoroscopic analysis of knee

368 arthroplasty kinematics during deep flexion kneeling. The Journal of arthroplasty 19(8): 998,

3692004

370 12. Komistek RD, Mahfouz MR, Bertin KC, Rosenberg A, Kennedy W. In vivo

371 determination of total knee arthroplasty kinematics: a multicenter analysis of an asymmetrical

372 posterior cruciate retaining total knee arthroplasty. The Journal of arthroplasty 23(1): 41,

3732008

374 13. Schmidt R, Komistek RD, Blaha JD, Penenberg BL, Maloney WJ. Fluoroscopic analyses

375 of cruciate-retaining and medial pivot knee implants. Clinical orthopaedics and related

376 research (410): 139, 2003

377 14. Wright J, Ewald FC, Walker PS, Thomas WH, Poss R, Sledge CB. Total knee

378 arthroplasty with the kinematic prosthesis. Results after five to nine years: a follow-up note. J

379 Bone Joint Surg Am 72(7): 1003, 1990

380 15. Hill PF, Vedi V, Williams A, Iwaki H, Pinskerova V, Freeman MA. Tibiofemoral

381 movement 2: the loaded and unloaded living knee studied by MRI. The Journal of bone and

382 joint surgery British volume 82(8): 1196, 2000

383 16. Iwaki H, Pinskerova V, Freeman MA. Tibiofemoral movement 1: the shapes and relative

384 movements of the femur and tibia in the unloaded cadaver knee. The Journal of bone and

385 joint surgery British volume 82(8): 1189, 2000

386 17. Komistek RD, Dennis DA, Mahfouz M. In vivo fluoroscopic analysis of the normal

387 human knee. Clinical orthopaedics and related research (410): 69, 2003 
18. Nakagawa S, Kadoya Y, Todo S, Kobayashi A, Sakamoto H, Freeman MA, Yamano Y.

Tibiofemoral movement 3: full flexion in the living knee studied by MRI. The Journal of bone and joint surgery British volume 82(8): 1199, 2000

19. Hoshino Y, Tashman S. Internal tibial rotation during in vivo, dynamic activity induces

392 greater sliding of tibio-femoral joint contact on the medial compartment. Knee surgery, sports

393 traumatology, arthroscopy : official journal of the ESSKA 20(7): 1268, 2012

20. Isberg J, Faxen E, Laxdal G, Eriksson BI, Karrholm J, Karlsson J. Will early

reconstruction prevent abnormal kinematics after ACL injury? Two-year follow-up using

21. Koo S, Andriacchi TP. The knee joint center of rotation is predominantly on the lateral

side during normal walking. Journal of biomechanics 41(6): 1269, 2008

22. Kozanek M, Hosseini A, Liu F, Van de Velde SK, Gill TJ, Rubash HE, Li G.

Tibiofemoral kinematics and condylar motion during the stance phase of gait. Journal of

402 biomechanics 42(12): 1877, 2009

403 23. Yamaguchi S, Gamada K, Sasho T, Kato H, Sonoda M, Banks SA. In vivo kinematics of 404 anterior cruciate ligament deficient knees during pivot and squat activities. Clinical 405 biomechanics (Bristol, Avon) 24(1): 71, 2009

24. Gustke K. Use of smart trials for soft-tissue balancing in total knee replacement surgery.

407 The Journal of bone and joint surgery British volume 94(11 Suppl A): 147, 2012

408 25. Roche M, Elson L, Anderson C. Dynamic Soft Tissue Balancing in Total Knee

409 Arthroplasty. Orthopedic Clinics of North America 45(2): 157, 2014

410 26. Gustke KA, Golladay GJ, Roche MW, Elson LC, Anderson CR. A New Method for

411 Defining Balance: Promising Short-Term Clinical Outcomes of Sensor-Guided TKA. The

412 Journal of arthroplasty 29(5): 955, 2014 
413 27. Gustke KA, Golladay GJ, Roche MW, Elson LC, Anderson CR. Primary TKA Patients

414 with Quantifiably Balanced Soft-Tissue Achieve Significant Clinical Gains Sooner than

415 Unbalanced Patients. Advances in Orthopedics 2014: 6, 2014

416 28. Gustke KA, Golladay GJ, Roche MW, Jerry GJ, Elson LC, Anderson CR. Increased

417 satisfaction after total knee replacement using sensor-guided technology. The bone \& joint

418 journal 96-b(10): 1333, 2014

419 29. Meneghini RM, Ziemba-Davis MM, Lovro LR, Ireland PH, Damer BM. Can

420 Intraoperative Sensors Determine the "Target" Ligament Balance? Early Outcomes in Total

421 Knee Arthroplasty. The Journal of arthroplasty 31(10): 2181, 2016

422 30. Noble PC, Scuderi GR, Brekke AC, Sikorskii A, Benjamin JB, Lonner JH, Chadha P,

423 Daylamani DA, Scott WN, Bourne RB. Development of a new Knee Society scoring system.

424 Clinical orthopaedics and related research 470(1): 20, 2012

425 31. Scuderi GR, Bourne RB, Noble PC, Benjamin JB, Lonner JH, Scott WN. The New Knee

426 Society Knee Scoring System. Clinical orthopaedics and related research 470(1): 3, 2012

427 32. Naal FD, Impellizzeri FM, Leunig M. Which is the Best Activity Rating Scale for

428 Patients Undergoing Total Joint Arthroplasty? Clinical orthopaedics and related research

429 467(4): 958, 2009

430 33. Dennis DA, Komistek RD, Mahfouz MR, Haas BD, Stiehl JB. Multicenter determination

431 of in vivo kinematics after total knee arthroplasty. Clinical orthopaedics and related research

432 (416): 37, 2003

433 34. Wasielewski RC, Galat DD, Komistek RD. Correlation of compartment pressure data

434 from an intraoperative sensing device with postoperative fluoroscopic kinematic results in

435 TKA patients. Journal of biomechanics 38(2): 333, 2005

436 
Table 1: Demographics in early lateral/late medial pivot kinematic pattern knees compared to knees with all other kinematic patterns

\begin{tabular}{|l|c|c|c|c|}
\hline & \multicolumn{2}{|c|}{ Kinematic Pattern } & & \\
\hline & $\begin{array}{c}\text { Early Lateral/Late } \\
\text { Medial Kinematic } \\
\text { Pattern }\end{array}$ & $\begin{array}{c}\text { All Other } \\
\text { Kinematic } \\
\text { Patterns }\end{array}$ & Statistic & $p$ \\
\hline $\mathrm{n}$ & 16 & 47 & & $\mathrm{t}=-0.16$ \\
\hline Mean age (in years) & 66.8 & 66.4 & 0.878 \\
\hline \% Female & 68.8 & 78.7 & $\mathrm{X}^{2}=0.419$ & 0.501 \\
\hline Mean BMI & 32.0 & 33.6 & $\mathrm{t}=0.84$ & 0.406 \\
\hline Median follow-up (in months) & 19.2 & 25.4 & $\mathrm{~W}=1642.0$ & 0.030 \\
\hline
\end{tabular}




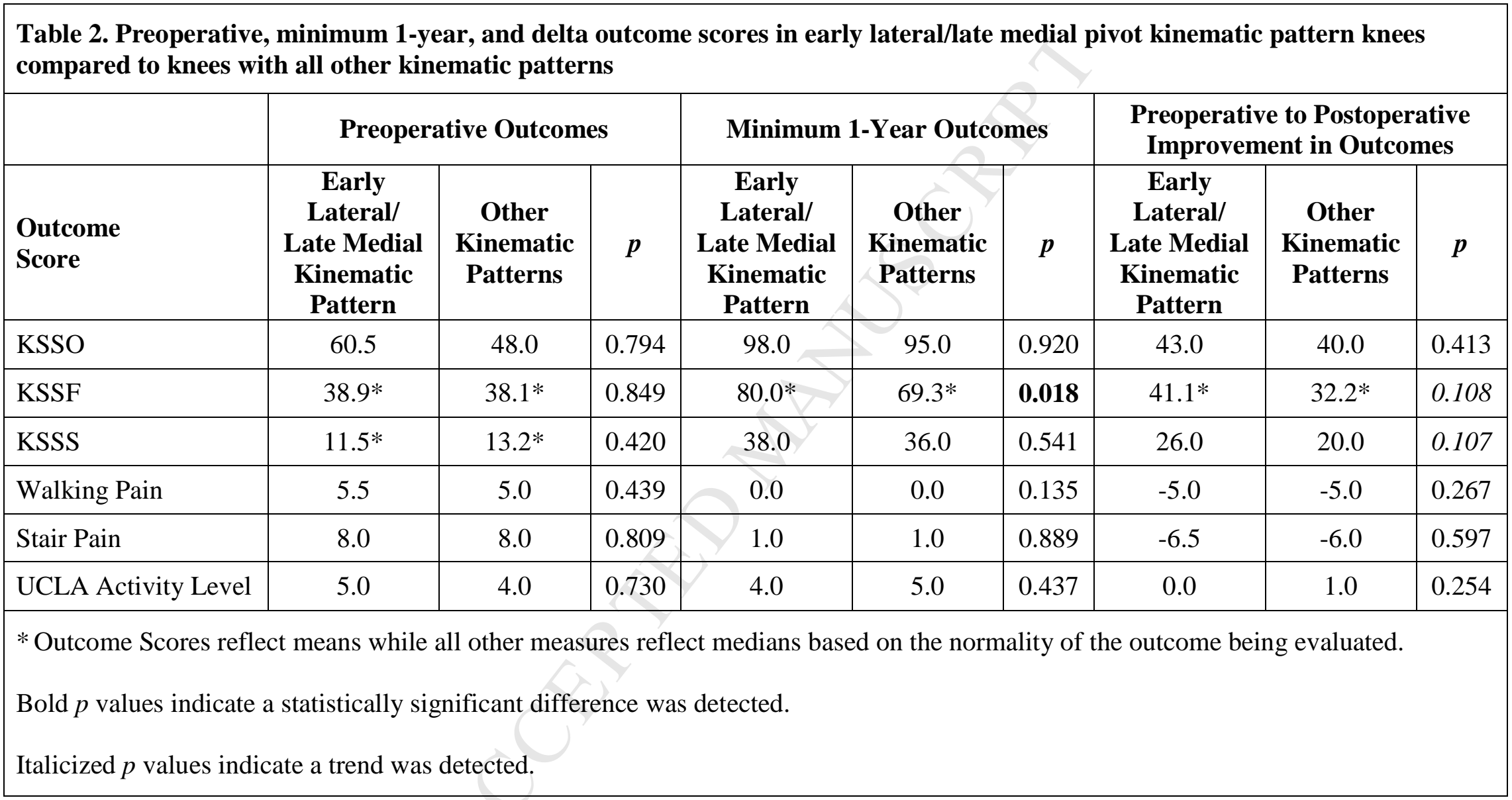




\begin{tabular}{|c|c|c|c|c|c|c|c|c|c|}
\hline \multirow[b]{2}{*}{$\begin{array}{l}\text { Outcome } \\
\text { Score }\end{array}$} & \multicolumn{3}{|c|}{ Preoperative Outcomes } & \multicolumn{3}{|c|}{ Minimum 1-Year Outcomes } & \multicolumn{3}{|c|}{$\begin{array}{l}\text { Preoperative to Postoperative } \\
\text { Improvement in Outcomes }\end{array}$} \\
\hline & LLM & MLL & $p$ & LLM & MLL & $p$ & LLM & MLL & $p$ \\
\hline KSSO & 68.0 & 43.5 & 0.061 & 98 & 95 & 0.640 & $31.6^{*}$ & $47.7 *$ & 0.077 \\
\hline KSSF & $39.3^{*}$ & $25.5^{*}$ & 0.086 & $87.5^{*}$ & $51.2 *$ & $<0.001$ & $48.3^{*}$ & $25.7 *$ & 0.008 \\
\hline KSSS & 8 & 10 & 0.844 & 40 & 33 & 0.043 & $27.5^{*}$ & $18.0^{*}$ & 0.022 \\
\hline Walking Pain & 4.5 & 5.5 & 0.793 & 0 & 1.5 & $* *$ & $-5.4 *$ & $-3.7 *$ & 0.323 \\
\hline Stair Pain & $7.1 *$ & $7.7 *$ & 0.665 & 0.5 & 2.5 & 0.220 & $-6.5^{*}$ & $-4.7^{*}$ & 0.207 \\
\hline UCLA Activity Level & 4.5 & 3.5 & 0.156 & $4.9 *$ & $3.7 *$ & 0.181 & 0 & 0 & 0.886 \\
\hline \multicolumn{10}{|c|}{$\begin{array}{l}\text { * Outcome Scores reflect means while all other measures reflect medians based on the nor } \\
\text { evaluated. }\end{array}$} \\
\hline \multicolumn{10}{|c|}{ ** Group medians could not be tested because all values for in the LLM group were zero. } \\
\hline \multicolumn{10}{|c|}{ Bold $p$ values indicate a statistically significant difference was detected. } \\
\hline \multicolumn{10}{|c|}{ Italicized $p$ values indicate a trend was detected. } \\
\hline
\end{tabular}




\section{ACCEPTED MANUSCRIPT}

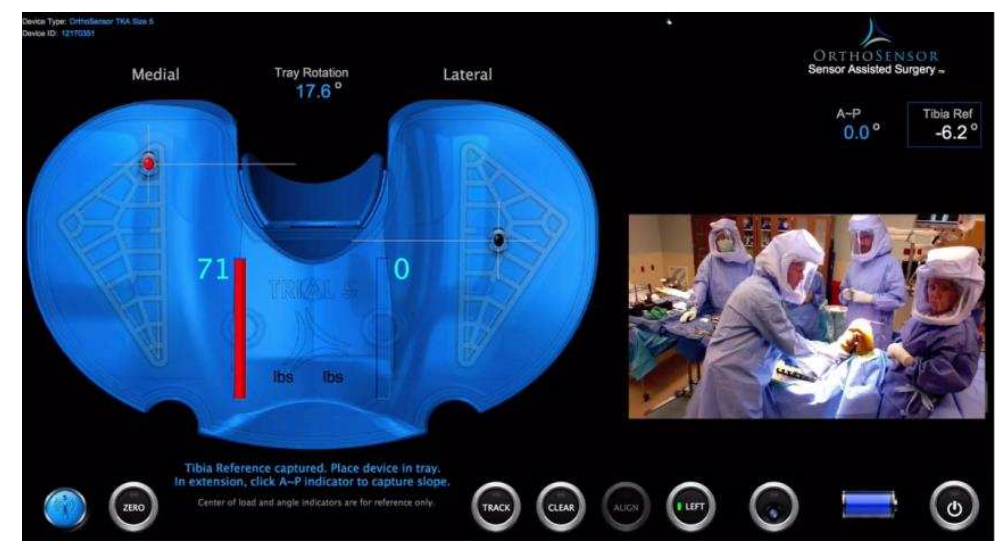




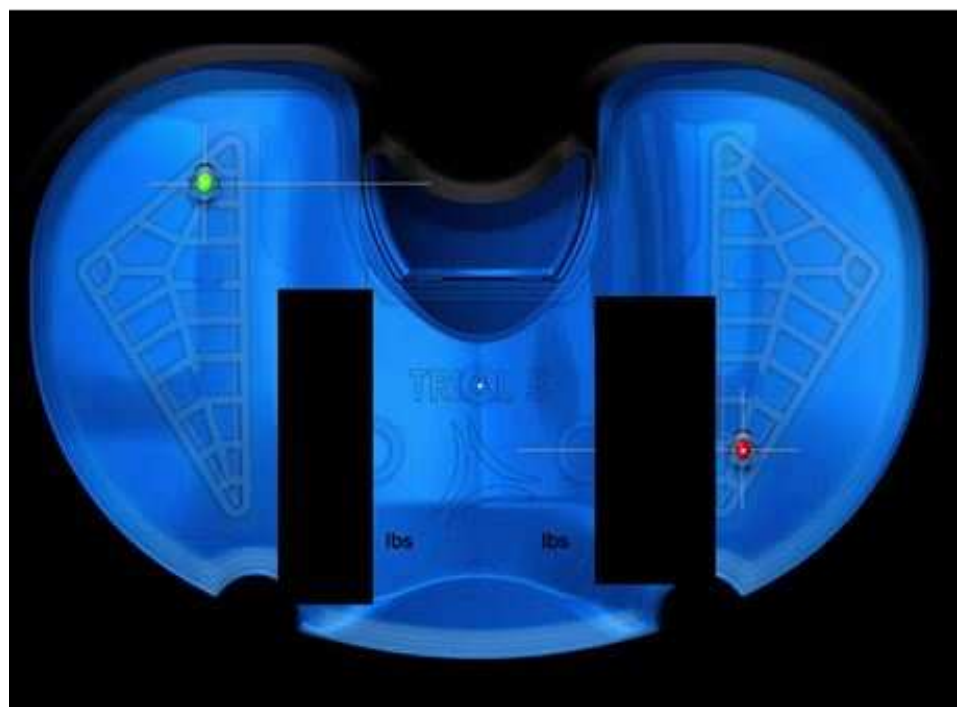



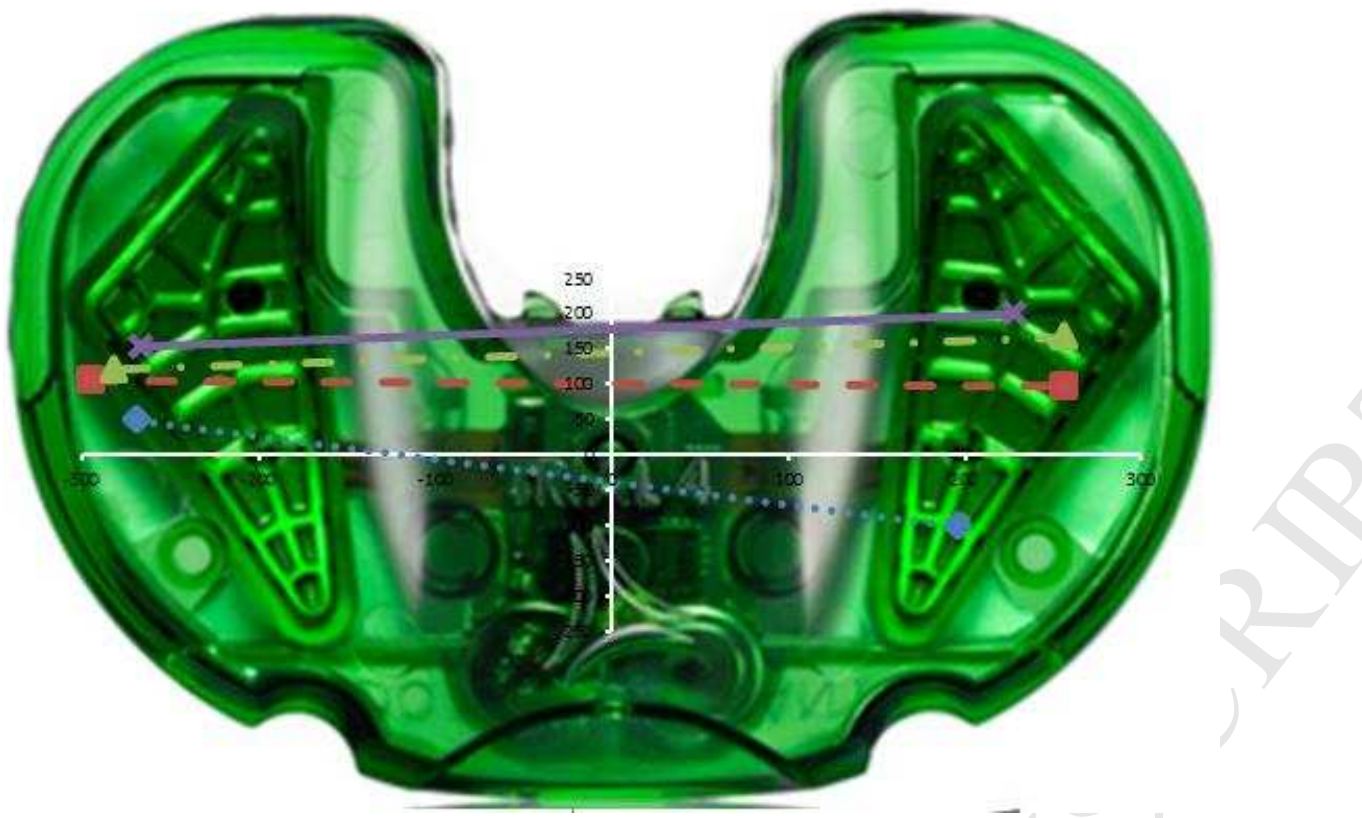


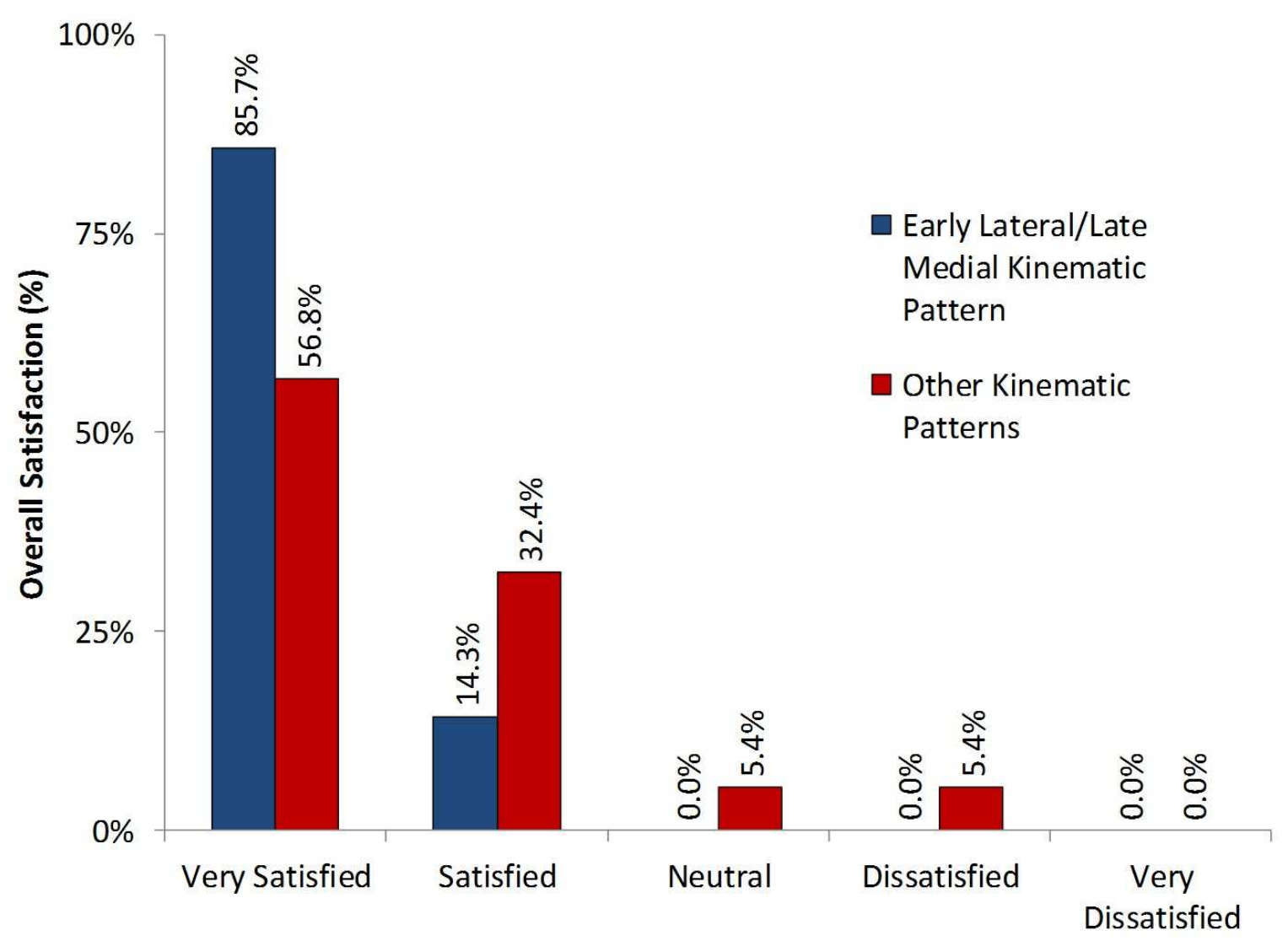




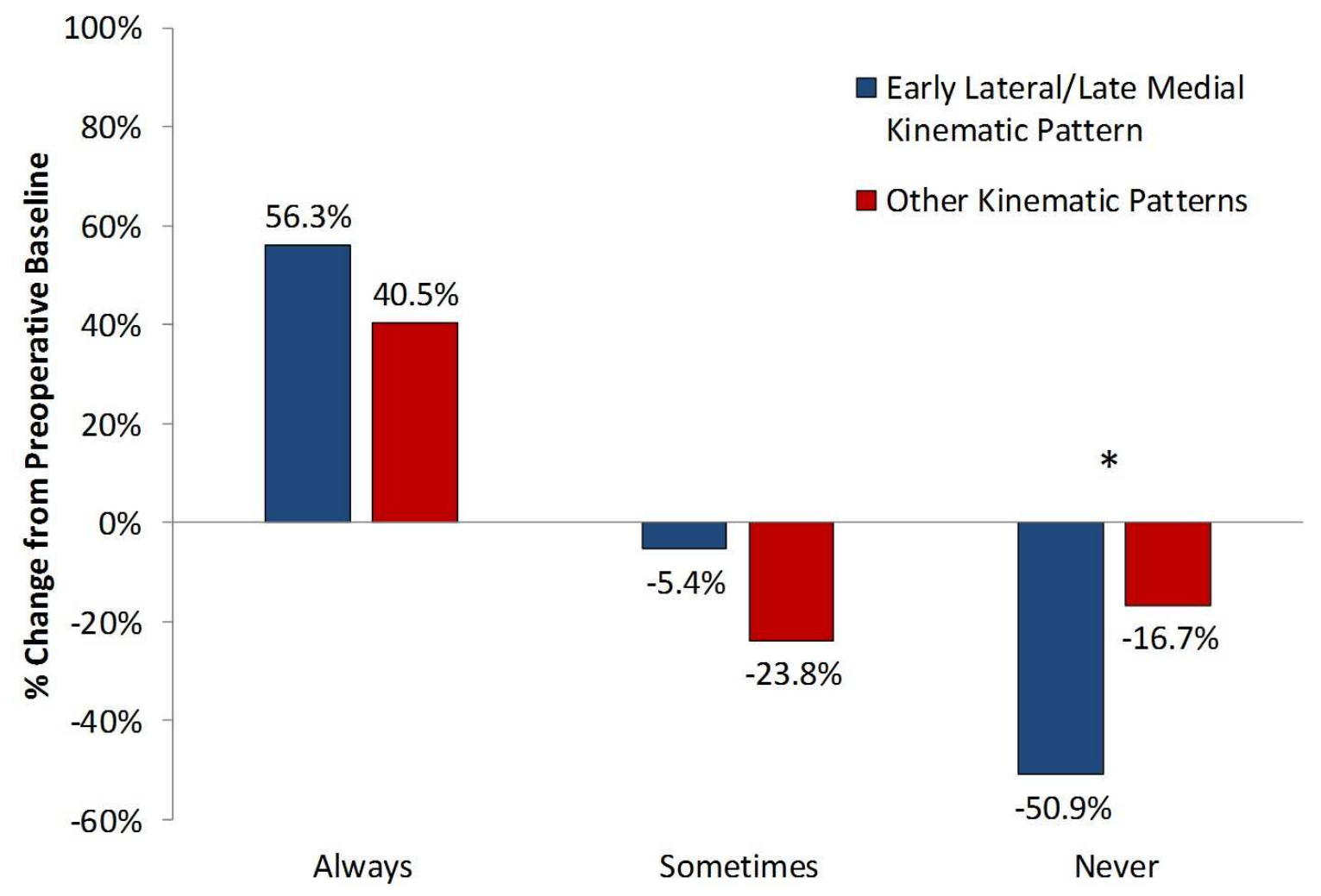




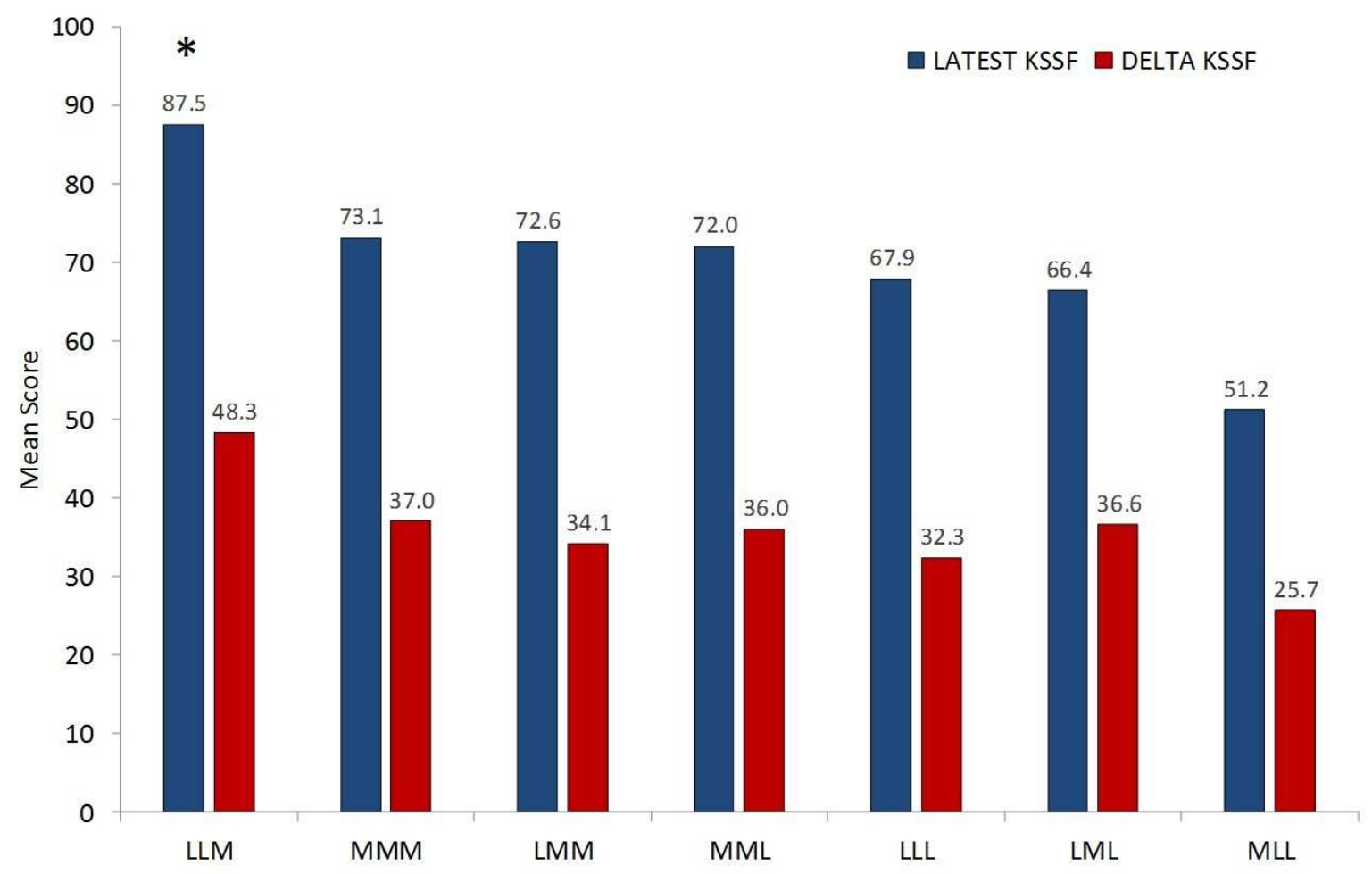




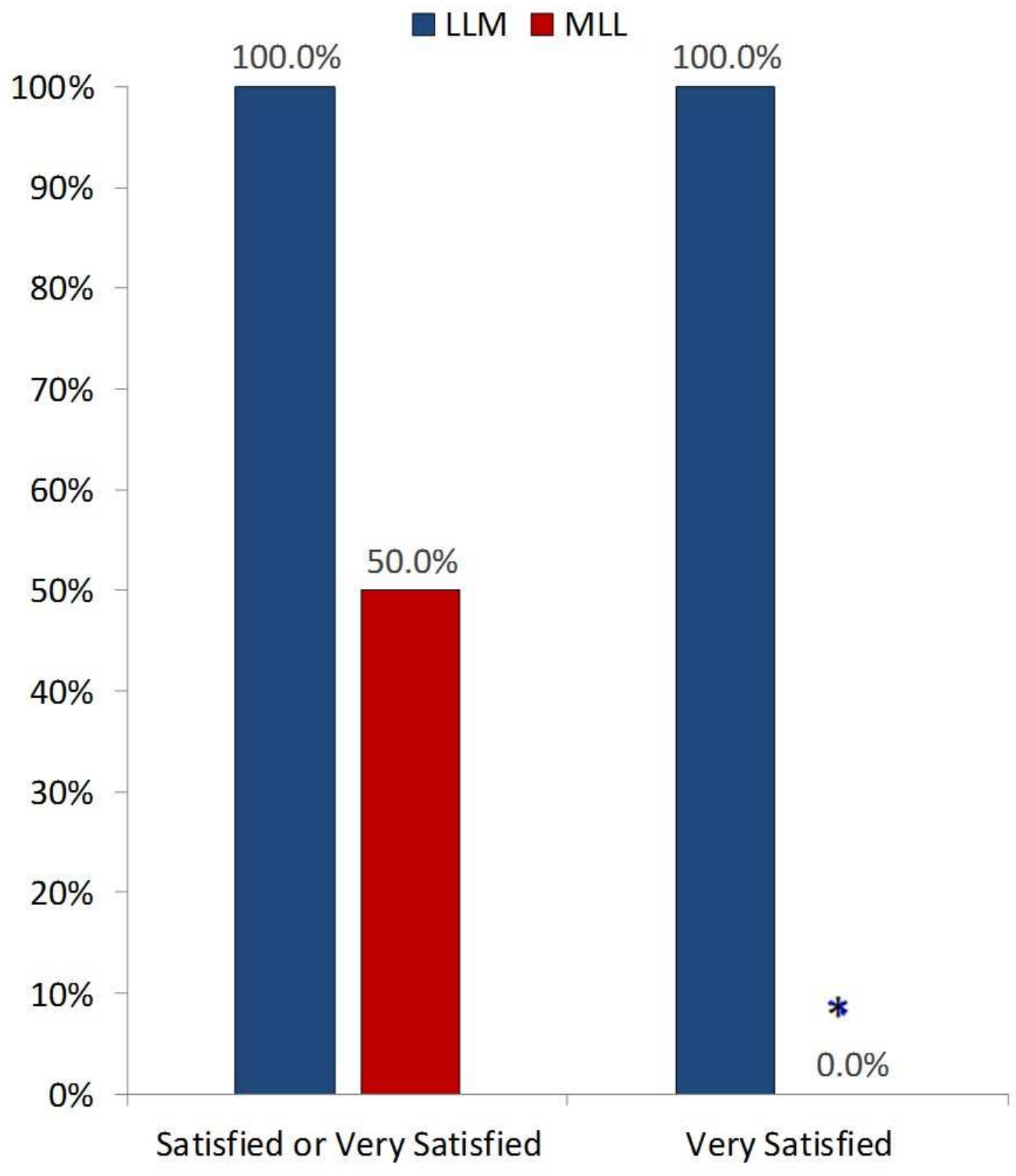

